Nervenarzt 2020 $\cdot 91: 518-529$ https://doi.org/10.1007/s00115-020-00919-8 Online publiziert: 11. Mai 2020 (c) Der/die Autor(en) 2020

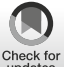

Andreas Ziegler' - Ekkehard Wilichowski ${ }^{2}$ Ulrike Schara ${ }^{3}$. Andreas Hahn ${ }^{4}$.

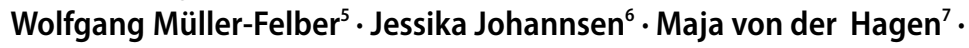
Arpad von Moers ${ }^{8} \cdot$ Corinna Stoltenburg $^{9} \cdot$ Afshin Saffari $^{1} \cdot$ Maggie C. Walter $^{10}$. Ralf A. Husain ${ }^{11}$. Astrid Pechmann ${ }^{12}$. Cornelia Köhler ${ }^{13}$. Veronka Horber ${ }^{14}$. Oliver Schwartz ${ }^{15} \cdot$ Janbernd Kirschner ${ }^{16}$

'Zentrum für Kinder- und Jugendmedizin Heidelberg, Sektion Neuropädiatrie und Stoffwechselmedizin, Universitätsklinikum Heidelberg, Heidelberg, Deutschland; ${ }^{2}$ Abteilung Neuropädiatrie und Sozialpädiatrie, Klinik für Kinder- und Jugendmedizin, Universitätsmedizin Göttingen, Göttingen, Deutschland; ${ }^{3}$ Abteilung für Neuropädiatrie, Zentrum für neuromuskuläre Erkrankungen des Kindesund Jugendalters, Universitätsklinikum Essen, Universität Duisburg-Essen, Essen, Deutschland; ${ }^{4}$ Abteilung Kinderneurologie, Sozialpädiatrie und Epileptologie, Zentrum Kinderheilkunde, Justus-LiebigUniversität, Gießen, Deutschland; ${ }^{5}$ Dr. v. Hauner'sche Kinderklinik, Universitätskinderklinik, LudwigMaximilians-Universität, München, Deutschland; ${ }^{6}$ Neuropädiatrie, Klinik und Poliklinik für Kinder- und Jugendmedizin, Universitätsklinikum Hamburg-Eppendorf, Hamburg, Deutschland; ' ${ }^{7}$ Abteilung Neuropädiatrie, Medizinische Fakultät Carl Gustav Carus, Technische Universität Dresden, Dresden, Deutschland; ${ }^{8}$ Klinik für Kinder- und Jugendmedizin, DRK Kliniken Berlin, Berlin, Deutschland; ${ }^{9}$ Sozialpädiatrisches Zentrum, Charité - Universitätsmedizin Berlin, Berlin, Deutschland; ${ }^{10}$ Friedrich-BaurInstitut, Neurologische Klinik und Poliklinik, Ludwig-Maximilians-Universität, München, Deutschland; ${ }^{11}$ Klinik für Neuropädiatrie, Universitätsklinikum Jena, Jena, Deutschland; ${ }^{22}$ Klinik für Neuropädiatrie und Muskelerkrankungen, Zentrum für Kinder- und Jugendmedizin, Universitätsklinikum Freiburg, Medizinische Fakultät, Albert-Ludwigs-Universität Freiburg, Freiburg, Deutschland; ${ }^{13}$ Abteilung für Neuropädiatrie mit Sozialpädiatrie, Klinik für Kinder- und Jugendmedizin, Ruhr-Universität Bochum, Bochum, Deutschland; ${ }^{14}$ Abteilung Neuropädiatrie, Entwicklungsneurologie und Sozialpädiatrie, Universitätsklinik für Kinder- und Jugendmedizin, Tübingen, Deutschland; ${ }^{15}$ Abteilung für Allgemeine Pädiatrie - Neuropädiatrie, Klinik für Kinder- und Jugendmedizin, Universitätsklinikum Münster, Münster, Deutschland; ${ }^{16}$ Abteilung Neuropädiatrie und Sozialpädiatrisches Zentrum, Zentrum für Kinderheilkunde, Universitätsklinikum Bonn, Bonn, Deutschland

\title{
Handlungsempfehlungen zur Gentherapie der spinalen Muskelatrophie mit Onasemnogene Abeparvovec - AVXS-101
}

\section{Konsensuspapier der deutschen Vertretung der Gesellschaft für Neuropädiatrie (GNP) und der deutschen Behandlungszentren unter Mitwirkung des Medizinisch- Wissenschaftlichen Beirates der Deutschen Gesellschaft für Muskelkranke (DGM) e.V.}

Die Genersatztherapie mit Onasemnogene Abeparvovec verspricht erstmals eine kausale Therapieoption für kleine Kinder mit spinaler Muskelatrophie (SMA) in Form einer einmaligen intravenösen Anwendung. Es liegen keine Langzeit- ergebnisse über die Nachhaltigkeit der Wirkung und möglicher unerwünschter Wirkungen inklusive potenzieller „Offtarget“-Effekte vor. Die Anwendung dieser neuen Therapieform muss daher unter strengen Sicherheitsauflagen und von entsprechend qualifizierten und erfahrenen neuromuskulären Behandlungszentren erfolgen. Die Wirksamkeit und Sicherheit der Behandlung und ein Vergleich gegenüber Nusinersen, der einzigen bisher zugelassenen Therapie, und 


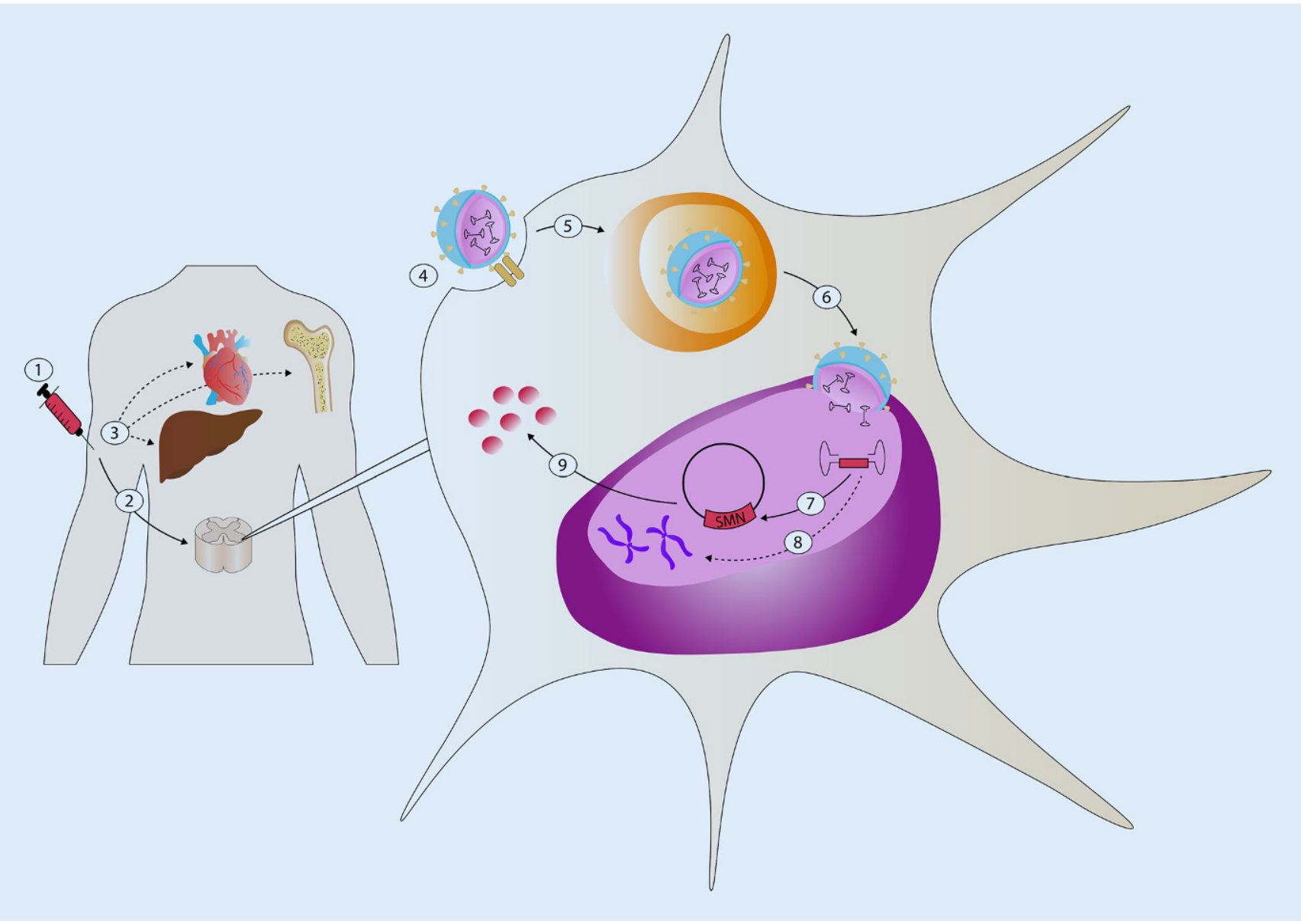

Abb. 1 A Wirkungsprinzip der AAV-basierten Gentherapie. (1) Intravenöse Applikation der AAV-basierten Gentherapie. (2) Ansteuerung des Zielgewebes, in diesem Falle der motorischen Vorderhornzellen im Rückenmark. (3) Off-target-Effekte durch Ansteuerung weiterer Gewebe wie der Leber (Transaminasenerhöhung), des Herzens (potenzielle Kardiotoxiziät) und des blutbildenden Systems (Blutbildveränderungen, insbesondere Thrombopenie). (4) Erkennung und Bindung von Oberflächenmolekülen des AAV-Kapsids durch glykosylierte Zelloberflächenrezeptoren der motorischen Vorderhornzelle und Internalisierung durch Endozytose. (5) Überführung der AAV in endosomal-lysosomale Abbauwege. (6) „Escape“ der AAV, Translokation an die Zellkernmembran und Verschmelzung des AAV-Kapsids mit der Zellkernmembran. (7) Freisetzung des selbstkomplementären Vektorgenoms in den Zellkern und Persistenz des Vektorgenoms mitsamt Transgen im Zellkern als ringförmiges extrachromosomales Episom. 8 In 0,1-1 \% der Fälle Integration des Vektorgenoms in die Wirts-DNA mit potenziellen Risiken wie einer malignen Transformation der transduzierten Zelle (insertionelle Mutagenese). (9) Expression des gewünschten Zielproteins durch das Transgen

ggf. im Verlauf anderen genmodulierenden Therapien, muss auch in der „Realworld“-Anwendung systematisch in einem wissenschaftlich fundierten, krankheitsspezifischen Register dokumentiert und evaluiert werden. Mit den neuen Therapien entstehen neue Herausforderungen an Diagnostik, Patientenversorgung und das Gesundheitssystem. Das vorliegende Konsensuspapier gibt konkrete Empfehlungen für die Anwendung dieser Gentherapie in der klinischen Praxis.

\section{Hintergrund}

Die spinale Muskelatrophie (SMA) ist mit einer Inzidenz von ca. 1 auf 7500 Lebendgeburten in Deutschland und einer Überträgerfrequenz von 1 zu 50 historisch die führende genetisch bedingte Ursache für Säuglingssterblichkeit [14]. Onasemnogene Abeparvovec (im Folgenden AVXS-101, nach Marktzulassung in Deutschland geplanter Handelsname Zolgensma $^{\circledR}$ ) ist ein Genersatztherapeutikum für die kausale Therapie der 5qassoziierten SMA, bei der es durch biallelische Mutationen im SMN1(Survival Motor Neuron1)-Gen zu einem Mangel an SMN-Protein in den $\alpha$-Motoneuronen kommt. Das SMN-Protein spielt eine grundlegende Rolle in der Regulation der Zellhomöostase und der Biogenese von Ribonukleoproteinen. Neuere Studien haben gezeigt, dass SMN-Protein auch an anderen „House-keeping“-Prozessen beteiligt ist, darunter mRNA-Trafficking und lokale Translation, Zytoskelettdynamik, Endozytose und Autophagie. Darüber hinaus hat sich gezeigt, dass das SMN-Protein Mitochondrien und bioenergetische Wege beeinflusst und die Funktion des Ubiquitin-ProteasomSystems reguliert [3]. Der Mangel an SMN-Protein führt durch fortschrei- 
Nervenarzt 2020 $\cdot 91: 518-529$ https://doi.org/10.1007/s00115-020-00919-8

(c) Der/die Autor(en) 2020

A. Ziegler · E. Wilichowski $\cdot$ U. Schara $\cdot$ A. Hahn $\cdot$ W. Müller-Felber $\cdot$ J. Johannsen $\cdot$ M. von der Hagen $\cdot$ A. von Moers $\cdot$ C. Stoltenburg $\cdot$ A. Saffari $\cdot$ M. C. Walter · R. A. Husain · A. Pechmann · C. Köhler · V. Horber · O. Schwartz · J. Kirschner

Handlungsempfehlungen zur Gentherapie der spinalen Muskelatrophie mit Onasemnogene Abeparvovec - AVXS-101. Konsensuspapier der deutschen Vertretung der Gesellschaft für Neuropädiatrie (GNP) und der deutschen Behandlungszentren unter Mitwirkung des MedizinischWissenschaftlichen Beirates der Deutschen Gesellschaft für Muskelkranke (DGM) e.V.

\section{Zusammenfassung}

Hintergrund. Die spinale Muskelatrophie (SMA) ist eine schwere, lebenslimitierende neurodegenerative Erkrankung. Seit Juli 2017 steht in Deutschland eine krankheitsmodifizierende und zugelassene Therapie mit Nusinersen zur Verfügung. Eine weitere vielversprechende Behandlungsmöglichkeit durch eine einmalige Applikation bieten konzeptionell Gentherapien. Im Mai 2019 wurde erstmals eine kausale Genersatztherapie zur Behandlung der spinalen Muskelatrophie von der U.S. Food and Drug Administration (FDA) zugelassen, die Zulassung in Europa ist beantragt.

Ziele. Dieses Konsensuspapier wurde auf Einladung der Deutschen Gesellschaft für Muskelkranke e. V. (DGM) unter Beteiligung der deutschen neuromuskulären Behandlungszentren, der deutschen Sektion der Gesellschaft für Neuropädiatrie (GNP) und unter Mitwirkung des Medizinisch-Wissenschaftlichen Beirates der DGM erarbeitet. Ziel ist es, die notwendigen Voraussetzungen für eine qualitätsgesicherte Anwendung der neuen Gentherapie zu definieren und die Grundlage für die Umsetzung in der klinischen Praxis zu schaffen.

Diskussion. Die Gentherapie mit Onasemnogene Abeparvovec besitzt das Potenzial, den Krankheitsverlauf der spinalen Muskelatrophie signifikant zu beeinflussen. Langzeitdaten über die Nachhaltigkeit der Wirkung und mögliche unerwünschte Wirkungen liegen derzeit noch nicht vor. Die
Anwendung dieser innovativen Therapieform muss in spezialisierten und entsprechend qualifizierten Behandlungszentren unter strengen Sicherheitsauflagen erfolgen. Die vorliegende Arbeit schlägt die hierfür notwendigen Rahmenbedingungen und Empfehlungen für die systematische Vorund Nachsorge unter Gentherapie vor. Wirksamkeit und Sicherheit der Therapie sollten in einem industrieunabhängigen, krankheitsspezifischen Register systematisch erfasst werden.

Schlüsselwörter

Spinale Muskelatrophie - Gentherapie . Zolgensma - Neuromuskuläre Erkrankungen . Konsensuspapier SMA

\section{Recommendations for gene therapy of spinal muscular atrophy with onasemnogene} abeparvovec-AVXS-101. Consensus paper of the German representatives of the Society for Pediatric Neurology (GNP) and the German treatment centers with collaboration of the medical scientific advisory board of the German Society for Muscular Diseases (DGM)

\begin{abstract}
Background. Spinal muscular atrophy (SMA) is a severe, life-limiting neurodegenerative disease. A disease-modifying and approved therapy with nusinersen has been available in Germany since July 2017. Gene therapies offer another promising treatment option through a once in a lifetime administration. In May 2019 a gene replacement therapy for the treatment of SMA was approved for the first time by the U.S. Food and Drug Administration (FDA). An application for approval in Europe has been submitted and is currently pending. Objective. This consensus paper was compiled at the invitation of the German Society for Muscular Diseases (DGM) with the participation of all potential German
\end{abstract}

neuromuscular treatment centers, the German section of the Society for Pediatric Neurology (GNP) and with the involvement of the medical scientific advisory board of the DGM. The aim was to define and establish the necessary prerequisites for a safe and successful application of the new gene replacement therapy in clinical practice. Conclusion. Gene replacement therapy with onasemnogene abeparvovec has the potential to significantly influence the course of SMA. Long-term data on sustainability of effects and possible adverse effects of gene replacement therapy are not yet available. The application of this innovative therapy must be carried out in specialized and appropriately qualified treatment centers under strict safety conditions. This article makes suggestions for the necessary framework conditions and gives recommendations for a systematic pretreatment and posttreatment assessment schedule under gene therapy. The effectiveness and safety of the therapy should be systematically documented in an industry-independent and disease-specific register.

\section{Keywords}

Spinal muscular atrophy - Gene therapy . Zolgensma - Neuromuscular diseases . Consensus paper SMA tende Degeneration der motorischen Vorderhornzellen ( $\alpha$-Motoneurone) im Rückenmark und Hirnstamm zu einer progressiven Muskelatrophie. AVXS101 ist ein Adeno-assoziierter viraler Vektor (AAV) der eine funktionstüchtige Kopie des humanen SMN1-Gens mit einem $\beta$-Aktin-Promotor enthält
(• Abb. 1; $[13,15])$. Das Genkonstrukt wird nach intravenöser Gabe über den Blutstrom verteilt und kann in den ersten 2 Lebensjahren die noch unreife Blut-Hirn-Schranke überwinden. Nach Erkennung und Bindung von Oberflächenmolekülen des AAV durch glykosylierte Zelloberflächenrezeptoren der motorischen Vorderhornzelle erfolgt die Internalisierung durch Endozytose. Es folgt die Überführung des AAV-Kapsids in endosomal-lysosomale Abbauwege und die Freisetzung des selbstkomplementären Vektorgenoms und des $S M N$-Transgens in den Zellkern. Im Zellkern persistiert das Vektorgenom 
mitsamt dem funktionsfähigen $S M N$ Transgen als ringförmiges extrachromosomales Episom. Eine Integration des Vektorgenoms in die Wirts-DNA ist theoretisch denkbar und wäre mit potenziellen Risiken wie einer onkogenen Transformation der transduzierten Zelle (sog. insertionellen Mutagenese) verbunden. Dieses Phänomen kann erst im Langzeitverlauf abschließend beurteilt und eingeschätzt werden $[10,12]$. Nach Gabe kommt es zu einer raschen Expression des gewünschten SMN-Zielproteins durch das Transgen. In-vitround In-vivo-Untersuchungen im Tiermodell ergaben eine stabile transgene Expression nach einmaliger Gabe, insbesondere in den $a$-Motoneuronen des Hirnstamms und Rückenmarks, aber auch in den Neuronen des motorischen Kortex. Früh appliziert zeigten tierexperimentelle Analysen im transgenen SMA-Tiermodell, dass die Motoneurone nicht nur erhalten bleiben, sondern dass diese auch eine normale morphologische Struktur und Funktion haben [5].

Neben der Wirkung in der Zielzelle müssen im kurzfristigen Intervall nach Gabe vor allem sog. „Offtarget“-Effekte berücksichtigt und regelmäßig überwacht werden. Diese fasst - Abb. 1 zusammen. Sie entstehen in erster Linie durch Ansteuerung weiterer Gewebe wie der Leber (Transaminasenerhöhung durch Abbau des AAV mit anschließender Antigenpräsentation und Aktivierung des Immunsystems an der Hepatozytenoberfläche), des Herzens (potenzielle Kardiotoxiziät wie z.B. Myokarditis mit Erhöhung von Troponinwerten) und des blutbildenden Systems (Blutbildveränderungen, insbesondere Thrombozytopenie; $[1,6])$.

Die Firma AveXis/Novartis legte im 2. Halbjahr 2018 die Daten der abgeschlossenen Phase-1/2-Studie (START; [8]) sowie die Zwischenauswertungen der noch laufenden Phase-3-Studien (STR1VE-US, STR1VE-EU, SPR1NT) zu Nebenwirkungen, Dosisfindung und Wirksamkeit bei mit AVXS-101 behandelten, <6 Monate alten SMA-Typ 1Patienten vor. Aufgrund dieser Daten wurde im Mai 2019 von der FDA die beschleunigte „Orphan-Drug“-Zulassung für die Behandlung von SMA-Kindern unter 2 Jahren mit biallelischen Mutationen im SMN1-Gen erteilt. Auch für den europäischen Bereich beantragte AveXis die Zulassung von AVXS-101. Momentan läuft das Zulassungsverfahren bei der europäischen Zulassungsbehörde (European Medicines Agency, EMA) noch. AVXS-101 ist das erste Genersatztherapeutikum für diese Indikation und in den USA mit einem Nettopreis von 2,1 Mio. US-Dollar für eine einmalige Applikation auch das bisher weltweit teuerste Medikament.

Wie bei anderen Medikamenten für seltene Erkrankungen sind die Daten in Bezug auf Sicherheit und Wirksamkeit zum Zeitpunkt der Zulassung noch äußerst begrenzt. Es ist deshalb unerlässlich, nach der Zulassung durch eine systematische Erfassung weitere Evidenzen zur langfristigen Wirksamkeit und Sicherheit der Therapie zu sammeln. Hierzu wurde in Deutschland von den DGM-zertifizierten Neuromuskulären Zentren das SMArtCARE-Register als krankheitsspezifische Datenbank für Patienten mit SMA etabliert (www. smartcare.de; [11]).

\section{Teilnehmer und Ablauf der Konsensusfindung}

Das vorliegende Konsensuspapier fasst die Ergebnisse von zwei unabhängig von der Industrie initiierten Konsensustreffen am 21.08.2019 und 18.11.2019 in Frankfurt/Main auf Einladung der Deutschen Gesellschaft für Muskelkranke e. V. (DGM) zusammen.

Angeschrieben wurden alle neuropädiatrischen Kliniken und Abteilungen und alle sozialpädiatrischen Zentren, die sich im Jahr 2017 für die Behandlung von Kindern und Jugendlichen mit SMA mit Nusinersen (Spinraza ${ }^{\circledR}$ ) bei der DGM registrieren ließen (www. dgm.org/muskelerkrankungen/spinalemuskelatrophie; Datei: Liste der mit Spinraza behandelnden Kliniken für Kinder). Während des ersten Treffens am 21.08.2019 wurden die Anforderungen definiert, die Behandlungszentren erfüllen müssen, um sich als Gentherapiezentrum zu qualifizieren. Des Weiteren wurden die prästationären, stationären und poststationären Proze- duren bestimmt, die für eine sichere und nachhaltige Therapie mit AVXS101 notwendig sind. Daraus definieren sich unmittelbar die strukturellen und personellen Anforderungen sowie der Finanzierungsaufwand zusätzlich zu den Kosten des Medikaments. Im Oktober 2019 wurde ein erster Entwurf an alle Teilnehmer versandt mit der Bitte um Kommentierung und Rückmeldung bis zum zweiten Treffen am 18.11.2019. An diesem Tag wurde der Text von den anwesenden Teilnehmern gesichtet und unter Berücksichtigung der zuvor eingegangenen Kommentare gewichtet. Nach der Finalisierung wurde der Text zwecks Freigabe nochmals an die federführenden Autoren versandt.

\section{Anforderungen an die Qualifikation der Behandlungs- einrichtung}

Die Besonderheiten der Therapie mit AVXS-101 (Neugeborene, Säuglinge und Kleinkinder mit einem potenziell großen Spektrum an klinischer Symptomatik und Verlaufsdynamik; innovatives Genersatzwirkprinzip mit einem viralen Vektor mit nur begrenzten Erfahrungen weltweit; hochpreisige Therapiekosten; Notwendigkeit einer mehrjährigen und komplexen Nachsorge; Monitoring auf unerwartete unerwünschte Wirkungen im Verlauf u.a.m.) stellen besonders hohe Anforderungen an die Struktur und die personelle Ausstattung der behandelnden Kliniken. Im Rahmen der Konsensusfindung wurden für die Behandlung von SMA-Patienten Kriterien formuliert, die ein Behandlungszentrum mindestens vorweisen muss, um die Anforderungen an die komplexe Vor- und Nachsorge unter Gentherapie mit AVXS-101 hinreichend erfüllen zu können. - Tab. 1 fasst die wichtigsten Empfehlungen zu den Struktur- und Personalvoraussetzungen zusammen. Gegebenenfalls sind weitere Vorgaben durch die zuständigen Behörden im Rahmen des Zulassungsverfahrens im Verlauf zu beachten.

Mit der Zulassung von AVXS-101 in den USA wird in mehrerer Hinsicht Neuland betreten [16]. Die hohen Kosten für ein einmal $\mathrm{zu}$ applizierendes Arz- 
Tab. 1 Konsensusempfehlungen für die fachlichen, organisatorischen, infrastrukturellen und personellen Voraussetzungen der Gentherapie-Behandlungszentren in Deutschland

(1) Umfangreiche Erfahrungen in der Behandlung der spinalen Muskelatrophie (SMA)

a. Allgemeine Erfahrungen in der Behandlung der SMA im Kindesalter, dokumentiert durch die Behandlung von $\geq 20$ Fällen mit dieser Diagnose (G12.0 oder G12.1) in der Behandlungseinrichtung innerhalb von 3 Jahren. Dies beinhaltet die interdisziplinäre Betreuung, Hilfsmittelversorgung und Beratung

b. $\quad$ Spezifische Erfahrung mit der medikamentösen Therapie der SMA, dokumentiert durch den Einsatz zur Behandlung der spinalen Muskelatrophie zugelassener Medikamente bei $\geq 15$ Fällen innerhalb von 3 Jahren, davon mindestens 5 mit Therapiebeginn im 1. Lebensjahr. Dokumentation der Therapieergebnisse durch regelmäßige Erhebung standardisierter Tests der motorischen Funktion

(2)

Personelle und fachliche Anforderungen an das Behandlungszentrum

a.

Anforderung an die Qualifikation des ärztlichen Personals

Die für die Behandlung von Kindern mit Onasemnogene Abeparvovec ärztlich verantwortliche Leitung und ihre Stellvertretung müssen Fachärzte für Kinder- und Jugendmedizin mit Schwerpunktbezeichnung Neuropädiatrie sein. Die ärztlich verantwortliche Leitung und ihre Stellvertretung müssen über eine mindestens zweijährige Berufserfahrung in einer Behandlungseinrichtung verfügen, die als Neuromuskuläres Zentrum der Deutschen Gesellschaft für Muskelkranke e. V. anerkannt ist. Bei Tätigkeit in Teilzeit kann die Tätigkeit in Relation zu einer Tätigkeit in Vollzeit angerechnet werden

b. $\quad$ Anforderungen an die Qualifikation der Physiotherapeuten

In der Behandlungseinrichtung müssen mindestens zwei Physiotherapeuten zur Verfügung stehen, die über mindestens zweijährige Erfahrungen in der physiotherapeutischen Diagnostik und Behandlung von Kindern mit neuromuskulären Erkrankungen verfügen. Sie müssen in der Durchführung standardisierter Muskeltests (entsprechend der Empfehlungen des SMArtCARE-Registers) geschult sein und praktische Expertise in der Anwendung der Testverfahren vorweisen können (z. B. im Rahmen klinischer Studien). Entsprechende Zertifikate als Nachweis liegen den behandelnden Kliniken vor

c. Verfügbarkeit eines psychosozialen Teams

Das Zentrum muss über Mitarbeiter aus den Bereichen Psychologie und Sozialpädagogik mit Erfahrung in der Betreuung von Patienten mit neuromuskulären Erkrankungen verfügen, um eine angemessene psychosoziale Versorgung der Kinder und ihrer Familien sicherzustellen

(3) Anforderungen an Infrastruktur und Organisation des Gentherapiezentrums

a. Das Behandlungszentrum erfüllt die Kriterien der von der DGM zertifizierten Neuromuskulären Zentren. Zum Nachweis der Kriterien reicht die Vorlage eines gültigen Zertifikats

b. Das Zentrum verfügt über eine stationäre Versorgungsmöglichkeit inkl. pädiatrischer Intensivmedizin mit Beatmungsplätzen für die multiprofessionelle Versorgung von Kindern mit neuromuskulären Erkrankungen

c. Es besteht eine SOP für die klinische, apparative und laborchemische Überwachung vor und nach der Behandlung mit Onasemnogene Abeparvovec

d. $\quad$ Eine ärztliche Betreuung gemäß Facharztstandard (Kinder- und Jugendmedizin) muss ununterbrochen für die stationäre Betreuung der Patienten, die mit Onasemnogene Abeparvovec behandelt wurden, verfügbar sein

e. $\quad$ Das Behandlungszentrum hält entsprechende Ressourcen vor, damit sich neu diagnostizierte Patienten mit spinaler Muskelatrophie mit einem Alter unter 2 Jahren innerhalb von 3 Werktagen nach Kontaktaufnahme mit dem Zentrum vorstellen können

f. Das Behandlungszentrum verfügt über eine eigene oder krankenhausversorgende Apotheke mit einer zertifizierten Abteilung zur Herstellung/Rekonstitution steriler Arzneimittel (Sterilabteilung). Es besteht eine SOP für die Herstellung/Rekonstitution steriler Arzneimitte nach biologischer Schutzstufe 1

g. Ambulante Nachsorge

i. Eine ärztliche Betreuung gemäß Facharztstandard (Kinder- und Jugendmedizin, Schwerpunkt Neuropädiatrie) muss werktäglich jederzeit für die ambulante Nachsorge der Patienten, die mit Onasemnogene Abeparvovec behandelt wurden, verfügbar sein. Eine Notfallambulanz muss kontinuierlich verfügbar sein

ii. Die unter 2b. genannten qualifizierten Physiotherapeuten und das unter 2c. genannte psychosoziale Team stehen auch im Rahmen der ambulanten Nachsorge zur Verfügung

h. Dokumentation

i. Die Behandlungseinrichtung beteiligt sich aktiv an dem SMArtCARE-Register und muss die personellen und strukturellen Voraussetzungen für den Einschluss der Patienten in das Register und ggf. andere erforderliche Dokumentationssysteme vorhalten. Insbesondere sind über einen Zeitraum von mindestens 5 Jahren zu dokumentieren:

1. Vortherapien

2. Unerwünschte Arzneimittelwirkungen

3. Dokumentation der motorischen Funktion durch standardisierte Tests

4. Dokumentation der respiratorischen Funktion, der Schluckfunktion und Sprachentwicklung

5. Gesamtüberleben 
neimittel erfordern neue Abrechnungsformen durch die Kostenträger. Weitere Kosten fallen an für die Vorbereitung der Therapie, den (theoretisch einmaligen) stationären Aufenthalt, aber vor allem für die anschließende ambulante Weiterbehandlung und Verlaufsuntersuchungen der behandelten Patienten über mindestens 5 Jahre. Dieses Konsensuspapier ist im Rahmen eines durch die DGM initiierten Treffens mit den VertreterInnen der neuropädiatrischen Kliniken und sozialpädiatrischen Zentren, die in Deutschland Kinder und Jugendliche mit SMA ambulant und stationär behandeln, konzipiert worden. Unter Berücksichtigung der aktuellen Empfehlungen des US-amerikanischen Zentrums für Gentherapie in Columbus, Ohio [2] fasst es die medizinisch notwendigen Voraussetzungen für eine verlässliche und sichere Therapie mit AVXS-101 inkl. der ambulanten Nachsorge zusammen und beziffert den dafür erforderlichen strukturellen, personellen, zeitlichen und finanziellen Aufwand. Unberührt von den hier vorgestellten Empfehlungen müssen nach erfolgter Zulassung die Informationen und Vorgaben der aktuell noch nicht vorliegenden Fachinformation zur Anwendung von AVXS-101 beachtet werden. Das Konsensuspapier ist ausdrücklich auch an die bei der Zulassung involvierten Gremien und Verbände adressiert. Die notwendige Vergütung des im Folgenden dargestellten Behandlungsalgorithmus wurde bereits im Deutschen Ärzteblatt vorgestellt [17].

\section{Praktische Durchführung der Therapie mit AVXS-101}

Die Therapie mit AVXS-101 setzt einen AAV9-Antikörper-Titer in einer Höhe von max. 1:50 voraus [2, 7]. Dieser muss im Vorfeld in einem Speziallabor bestimmt werden. Rund $5 \%$ aller rekrutierten SMA-Kinder konnten aufgrund erhöhter AAV-Titer an den klinischen Studien zu AVXS-101 nicht teilnehmen. Die Therapie erfolgt nach Ausschluss von Kontraindikationen stationär unter einer fortlaufenden Prednisolontherapie, die $24 \mathrm{~h}$ vor Gabe begonnen sein, über mindestens 30 Tage durchgeführt und dann langsam ausgeschlichen werden muss. Etwa ein Drittel aller behandelten Kinder zeigte in den klinischen Studien zwar asymptomatische, teilweise aber erhebliche Erhöhungen der Lebertransaminasen bis über das 20-Fache der oberen Normgrenzen. Diese erforderten in den Studien oftmals eine Verlängerung, gegebenenfalls auch eine Intensivierung der immunsuppressiven Therapie. Ein individuelles Vorgehen wird damit notwendig. Die Erfahrungen aus den klinischen Studien zeigen, dass die ambulante Nachbeobachtung der Patienten wegen potenzieller Nebenwirkungen, aber insbesondere auch zur Erfassung der gewünschten Wirkungen umfassend, anfangs engmaschig und vor allem langfristig sein muss. Sowohl im Rahmen der sog. STR1VE-US Studie als auch in der europäischen STR1VEEU-Studie traten nach Gentherapie mit AVXS-101 jeweils ein Todesfall auf, beide wurden als nicht behandlungsbezogen eingestuft. Allerdings werden unerwünschte Ereignisse wie erhöhte Transaminasen, Thrombozytopenie sowie Hypotonie des Blutdrucks, die zeitlich mit Atemwegsinfektionen in Verbindung stehen, als nicht vollkommen auszuschließende Ereignisse im Zusammenhang mit der Anwendung von AVXS-101 eingeschätzt.

Die notwendigen und im Konsensus vorgeschlagenen Untersuchungen für die ambulante Vorbereitung, stationäre Gabe und anschließende ambulante Nachbetreuung der Kinder mit SMA unter Gentherapie mit AVXS-101 fasst - Tab. 2 zusammen. Zur besseren Übersicht werden drei wesentliche Phasen der spezialisierten Betreuung unterschieden: (1) die prästationäre Phase mit entsprechender Vorbereitung, Indikationsstellung und Beantragung der Gentherapie, (2) die stationäre Phase mit der tatsächlichen Gabe und entsprechend engmaschiger Überwachung und (3) die wichtige Phase der poststationären Langzeitnachbeobachtung nach erfolgter Gentherapie.

\section{Prästationäre Phase (Aufklärung, Planung und Beratung)}

Der erstmalige Kontakt mit dem spezialisierten Neuromuskulären Zentrum sollte ohne Zeitverzug bereits beim ersten Verdacht auf das Vorliegen einer SMA erfolgen. Alternativ kann zum Ausschluss bzw. zur Bestätigung der Diagnose direkt in der kinderärztlichen Praxis eine molekulargenetische Diagnostik auf das Vorliegen einer spinalen Muskelatrophie eingeleitet werden. Viele humangenetische Labore bieten die MLPA-Analytik auf das Vorliegen einer SMA an. In den Bundesländern, in denen bereits das Pilotprojekt für das SMA-Neugeborenenscreening läuft, erfolgt bei positivem Befund eine direkte Übergabe an das spezialisierte Behandlungszentrum. Ansonsten halten die akkreditierten Behandlungszentren entsprechende Ressourcen vor, damit sich neu diagnostizierte Patienten mit SMA unter 2 Jahren innerhalb von 3 Werktagen nach Kontaktaufnahme mit dem Zentrum vorstellen können, um die Therapieeinleitung und Beratung der Familie umgehend einzuleiten. Die Indikationsstellung und Überwachung der Therapie muss durch einen Facharzt für Kinder- und Jugendmedizin mit Schwerpunkt Neuropädiatrie und mehrjähriger Erfahrung in der Betreuung neuromuskulärer Patienten erfolgen. Wichtige Bestandteile der dann folgenden Beratung der Familien und Planung des weiteren Vorgehens sind in - Tab. 2 dargestellt. Folgende Aspekte sollten unbedingt Bestandteil der Beratung, der vorbereitenden Diagnostik und Aufklärung sein:

- Falls bis zu diesem Zeitpunkt noch nicht erfolgt, erfolgt die sofortige Einleitung einer molekulargenetischen Diagnostik (MLPA) auf das Vorliegen einer SMA inklusive Bestimmung der SMN2-Kopienzahl in entsprechend akkreditierten humangenetischen Laboratorien.

- Ausführliche Aufklärung über das Krankheitsbild SMA, über die zur Verfügung stehenden Therapiemöglichkeiten mit einer objektiven $\mathrm{Ab}$ wägung des individuell besten Vorgehens im Hinblick auf die Auswahl des Präparates und den zeitlichen 


\section{Konsensuspapiere}

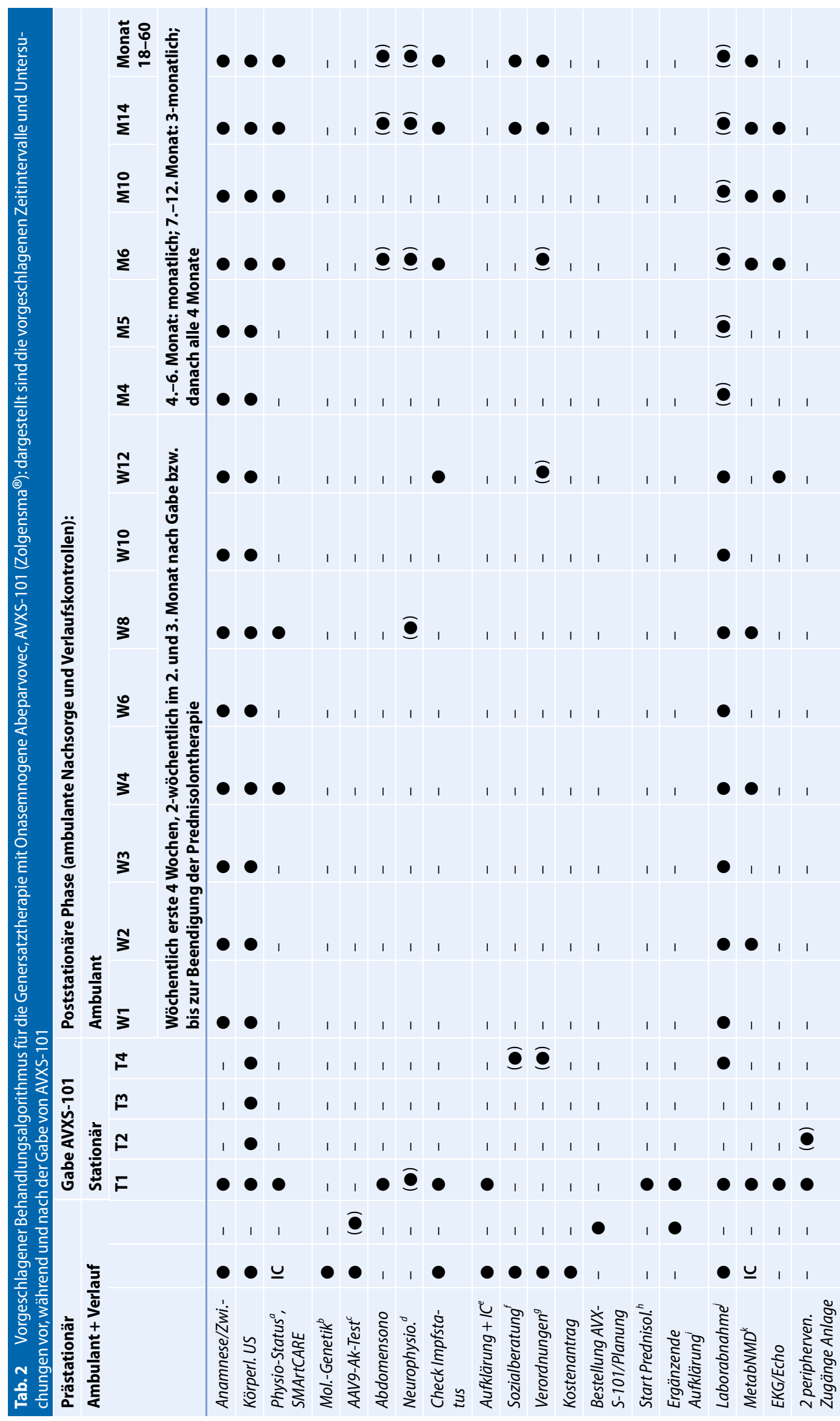




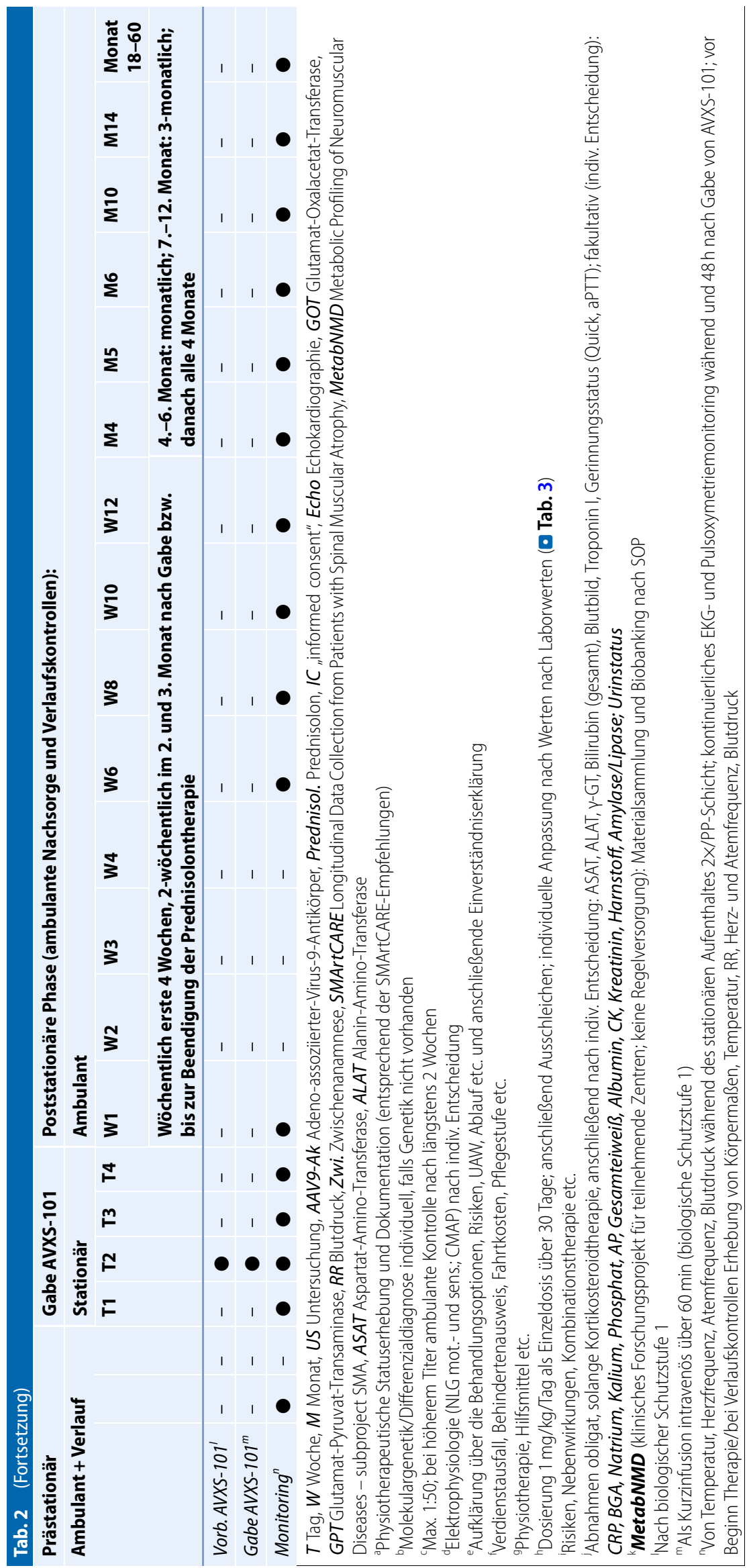

Ablauf der geplanten Untersuchungen (symptomatisch, kausal: SMN2Genenhancing vs. SMN1-Genersatztherapie).

- Aufklärung und Einschluss in das SMArtCARE-Projekt zur longitudinalen Sammlung erforderlicher Evidenzdaten.

- Standardisierte motorische Funktionsdiagnostik gemäß den SMArtCARE-Empfehlungen.

- Vorbereitende Diagnostik (• Tab. 2). Die Untersuchungen können nach Maßgabe des Zentrums auch im stationären Rahmen erfolgen.

- Überprüfung des Impfstatus: Impfungen nach STIKO-Empfehlungen und saisonale Impfungen bis eine Woche vor Beginn der Gentherapie (Patient und ggf. Bezugspersonen) - die aktualisierten Empfehlungen sowie vorgeschlagene Sicherheitsabstände vor und nach Gentherapie bzw. unter Prednisolontherapie müssen der zukünftigen deutschen Fachinformation des Herstellers entnommen werden.

- Für betroffene Kinder unter 2 Jahren wird in den Wintermonaten eine RSV-Prophylaxe mit Palivizumab empfohlen (für alle „non-sitters“).

- Einleitung einer multidisziplinären Betreuung und Beratung der Familien inklusive Sozialberatung (Schwerbehindertenausweis, Pflegegrad, Fahrkostenerstattung u. a.), Verordnung von Physiotherapie und ggf. Hilfsmitteln, orthopädische, pneumologische Anbindung und Ernährungsberatung, ggf. palliativmedizinische Exploration und Schlafmedizin $[4,9]$.

- Nach Indikationsstellung erfolgt die Sicherstellung der Kostenübernahme durch den Kostenträger.

- Im Vorfeld der stationären Aufnahme muss eine Evaluation des AAV9Titers (Dauer der Bestimmung etwa 5 Werktage) erfolgen: Dieser darf maximal 1:50 betragen; bei höherem Titer wird eine einmalige Kontrolle nach frühestens 2 Wochen empfohlen. Bei Neugeborenen und jungen Säuglingen kann auch ein erhöhter maternaler Titer vorliegen. In diesem Fall kann individuell eine weitere 
AAV9-Titer-Verlaufskontrolle in Abhängigkeit von der Symptomatik erwogen werden.

- Es wird ein professionelles Case-Management oder die Betreuung durch Patientenlotsen an den jeweiligen Standorten empfohlen. Im Rahmen des komplexen Planungs- und Nachsorgeprozesses brauchen die Familien einen verlässlichen Ansprechpartner, der die anstehenden Termine und die multidisziplinäre Betreuung der Familien organisiert.

\section{Stationäre Phase (Durch- führung der Therapie mit AVXS-101)}

Nach erfolgter Indikationsstellung für die Gentherapie durch das spezialisierte Neuromuskuläre Zentrum, Ausschluss von Kontraindikationen, vorliegender schriftlicher Kostenübernahmeerklärung durch die Krankenkasse und ausführlicher Aufklärung der Familien mit Einholung einer schriftlichen Einverständniserklärung erfolgt die stationäre Aufnahme im Behandlungszentrum. Der stationäre Aufenthalt sollte folgende wesentliche Aspekte beinhalten und entsprechend im Vorfeld geplant werden (• Tab. 2):

- Jedes Behandlungszentrum besitzt eine schriftliche Standardarbeitsanweisung (SOP) zur Planung und Durchführung der intravenösen Genersatztherapie mit AVXS-101. Diese wird regelmäßig aktualisiert, mindestens alle 12 Monate. Die Inhalte der Konsensusempfehlungen sowie zukünftige Vorgaben der Fachinformation sind darin implementiert.

- Bestellung von AVSX-101 durch den betreuenden Arzt und die Klinikapotheke im Vorfeld der stationären Aufnahme. Empfehlungen für den Bestellprozess und regelmäßig aktualisierte Formulare werden vom Hersteller Avexis zur Verfügung gestellt.

- Die stationäre Unterbringung soll entsprechend den lokalen Hygienerichtlinien bezüglich des Umgangs mit Erregern, die der biologischen Schutzstufe 1 entsprechen, erfolgen. Es sollte vermieden werden, dass andere Patienten mit SMA, die möglicherweise für eine Therapie zu einem späteren Zeitpunkt in Betracht kommen, oder akut infektiöse Patienten in unmittelbarer Nähe untergebracht sind. Nach Maßgabe des lokalen Behandlungszentrums kann ggf. eine Aufnahme auf eine pädiatrische Intensivstation erwogen werden.

- Tag 1 des stationären Aufenthaltes:

- Beginn der enteralen (oral oder via Sondierung) Einnahme von Prednisolon ( $1 \mathrm{mg} / \mathrm{kg} / \mathrm{Tag})$ als Einzeldosis $24 \mathrm{~h}$ vor AVXS-101-Therapie, Fortsetzung über mindestens 30 Tage, dann Ausschleichen nach Maßgabe der pädiatrischen Endokrinologie (Näheres siehe • Tab. 3); ein begleitender Magenschutz, z. B. mit Esomeprazol, wird empfohlen.

- Gegebenenfalls Wiederholung oder Erstbefund der motorischen Funktionsdiagnostik (entsprechend der SMArtCARE-Empfehlungen).

- EKG, Echokardiographie und weitere Diagnostik (• Tab. 2).

- Tag 2 des stationären Aufenthaltes:

- Legen von 2 peripher-venösen Zugängen.

- Gewichtsadaptierte Zubereitung des Zolgensma ${ }^{\circledR}$-Präparates in der Klinikapotheke entsprechend der Fachinformation und den Vorgaben des Herstellers, Transport zum Patienten; das Präparat ist nach Zubereitung bei Raumtemperatur $8 \mathrm{~h}$ haltbar.

- Intravenöse Infusion von AVSX101 über 60 min durch geschulte MitarbeiterInnen und unter Beachtung der biologischen Schutzstufe 1 („biosafety level 1“ [BSL] nach EURichtlinie 2000/54/EG und Biostoffverordnung) entsprechend der Fachinformation.

- Engmaschiges Monitoring der Patienten (• Tab. 2).

- Benutzen von Handschuhen durch Betreuungspersonen beim Windeln (Stuhlgang) bis 30 Tage nach der Infusion (besonders wichtig für Schwangere).

- Tag 3 und Tag 4 des stationären Aufenthaltes:
- Es wird eine stationäre Überwachung der Kinder von mindestens $48 \mathrm{~h}$ nach Applikation der Gentherapie empfohlen.

- In dieser Zeit Monitoring von klinischem Status, Temperatur, Herzfrequenz, Atemfrequenz, Blutdruck und EKG- und Pulsoxymetriemonitoring.

- Bei komplikationslosem Verlauf kann eine Entlassung frühestens $48 \mathrm{~h}$ nach Gabe von AVXS-101 erfolgen. Am Entlasstag werden die ambulanten Folgeuntersuchungen geplant und koordiniert.

\section{Poststationäre Phase (ambulante Nachsorge und Verlaufskontrollen)}

In den ersten 12 Monaten nach der Gentherapie wird eine engmaschige Anbindung an das spezialisierte Behandlungszentrum dringend vorausgesetzt. Die Nachsorge erfolgt nach der Darstellung in - Tab. 2 wöchentlich in den ersten 4 Wochen, 2-wöchentlich im 2. und 3. Monat nach der Gentherapie bzw. bis zur Beendigung der Prednisolontherapie. Bei unkompliziertem Verlauf kann die Nachsorge ab Monat 12 nach der Gentherapie auf ambulante Kontrollen alle 4 Monate gestreckt werden. Es wird eine standardisierte Nachsorge über mindestens 5 Jahre nach erfolgter Genersatztherapie mit regelmäßigen und systematischen ambulanten Nachkontrollen empfohlen. Der Algorithmus der vorgeschlagenen Untersuchungen und der standardisierten Befunddokumentation wird in - Tab. 2 zusammengefasst. Auch nach Ablauf der 5 Jahre müssen die Patienten im den neuromuskulären Spezialzentren mindestens 2-mal jährlich gesehen und systematisch nachkontrolliert werden.

\section{Empfehlungen zur Immunsuppression}

In der amerikanischen Fachinformation wird eine begleitende orale Prednisolontherapie zur Vermeidung einer überschießenden Immunreaktion in einer Dosierung von $1 \mathrm{mg} / \mathrm{kgKG} / \mathrm{Tag}$ empfohlen. Diese muss $24 \mathrm{~h}$ vor der Gabe 
Tab. 3 Konsensusempfehlungen zur oralen Prednisolontherapie zur individuellen Prophylaxe von AVXS-101-assoziierten immunologischen Off-target-Nebenwirkungen. Dargestellt sind die offiziellen Vorgaben der Fachinformation, ergänzt von den individuellen Anpassungsvorschlägen der Konsensus-Gruppe in verschiedenen Ausgansszenarien

\section{Orale Prednisolontherapie}

Zum Beispiel (Decortin-H-Tabletten [bedingt suspendierbar] oder Prednihexal/Prednisolon AL [suspendierbar nach Herstellerangabe]) inkl. Magenschutz, z. B. mit Esomeprazol

\begin{tabular}{|c|c|}
\hline Standard: & $\begin{array}{l}\text { Beginn } 24 \mathrm{~h} \text { vor AVXS-101-Gabe bis mindestens } 30 \text { Tage danach; tägliche Einnahme morgens vor dem Essen in einer } \\
\text { Einzeldosis }\end{array}$ \\
\hline Regelfall: & $\begin{array}{l}\text { Bei unkompliziertem Verlauf und ASAT/ALAT-Werten <2-fach des oberen Normwertes nach } 30 \text { Tagen: Ausschlei- } \\
\text { chen über } 28 \text { Tage mit wöchentlicher Reduktion der Dosis um ein Viertel der Gesamtdosis und ggf. Umstellung auf } \\
\text { Hydrokortison nach Maßgabe der lokalen Abteilung für pädiatrische Endokrinologie }\end{array}$ \\
\hline Ausnahmefall 1: & $\begin{array}{l}\text { Bei ASAT/ALAT-Werten >2-fach des oberen Normalwertes nach den ersten } 30 \text { Tagen: Fortsetzung der Therapie in } \\
\text { unveränderter Dosierung ( } 1 \mathrm{mg} / \mathrm{kgKG} / \mathrm{Tag} \text { ) mit weiterhin wöchentlichen Laboruntersuchungen bis ASAT/ALAT-Werte } \\
<2 \text {-fach des oberen Normalwertes (ggf. Dosisanpassung siehe oben), dann Ausschleichen über } 28 \text { Tage (siehe unter } \\
\text { Regelfall) }\end{array}$ \\
\hline Ausnahmefall 2: & $\begin{array}{l}\text { Falls in den ersten } 4 \text { Wochen nach Gabe von AVXS-101 die Transaminasen auf }>400 \mathrm{U} / \mathrm{L} \text { (entspricht } 6,67 \mu \mathrm{mol} /[\mathrm{s} \bullet \mathrm{L}] \\
\text { in SI-Einheiten) ansteigen, wird eine Erhöhung der Steroiddosis auf } 2 \mathrm{mg} / \mathrm{kgKG} / \mathrm{Tag} \text { für mindestens } 1 \text { Woche empfoh- } \\
\text { len, bis Transaminasen mindestens halbiert, dann wieder auf } 1 \mathrm{mg} / \mathrm{kg} \text { reduzieren und die Therapiedauer verlängern, } \\
\text { bis der Regelfall eintritt }\end{array}$ \\
\hline Ausnahmefall 3: & $\begin{array}{l}\text { Falls in den ersten } 4 \text { Wochen nach Gabe von AVXS-101 die Transaminasen auf }>1000 \mathrm{U} / \mathrm{L} \text { (entspricht } 16,67 \mu \mathrm{mol} /[\mathrm{s} \bullet \\
\text { L] in SI-Einheiten) ansteigen, wird Rücksprache mit der Kommission „neue Therapien“ der Gesellschaft für Neuropä- } \\
\text { diatrie (GNP) bzw. der Firma empfohlen, ggf. mit daraus resultierender Empfehlung zur intravenösen Pulstherapie } \\
\text { mit Methylprednisolon in einer Dosierung von } 20 \mathrm{mg} / \mathrm{kgKG} / \text { Tag i.v. über } 3 \text { Tage, bis Ausnahmefall } 2 \text { bzw. } 1 \text { eintritt; } \\
\text { alternativ: Erweiterung der immunmodulatorischen Therapie nach individueller Entscheidung }\end{array}$ \\
\hline
\end{tabular}

von AVXS-101 begonnen und mindestens 30 Tage verabreicht werden. Anschließend empfiehlt der Hersteller eine individuelle Anpassung bzw. ein langsames Ausschleichen des Prednisolons in Abhängigkeit von der Höhe der hepatischen Transaminasen. Die Empfehlungen aus der amerikanischen Fachinformation sind in - Tab. $3 \mathrm{zu}$ sammengefasst. In der täglichen Praxis waren aber bei den wenigen bisher in Deutschland therapierten Patienten individuelle Anpassungen der Steroiddosis bereits in kurzem Abstand zur Gabe erforderlich. - Tab. 3 stellt daher denkbare Ausnahmefälle aus der klinischen Praxis dar mit entsprechenden Empfehlungen zur individuellen Titration und Anpassung der Prednisolondosis. Im Falle einer wiederholten und persistierenden Erhöhung der Troponin-IWerte wird eine vorgezogene und erweiterte kardiale Diagnostik nach Maßgabe und in Rücksprache mit der lokalen Abteilung für pädiatrische Kardiologie empfohlen. Der Ausschleichprozess des Prednisolons erfordert in Abhängigkeit von der Behandlungsdauer ein individuelles Reduktionsschema der Kortikoide und eine Umstellung auf Hydrokortison nach Maßgabe und in Rücksprache mit den lokalen Abteilungen für pädiatrische Endokrinologie.

Die dargestellten Empfehlungen stellen das Resultat des Konsensusprozesses dar, sie sind nicht in der bisher verfügbaren amerikanischen Fachinformation enthalten. Beim Auftreten nichthepatischer systemischer Nebenwirkungen muss eine Therapie entsprechend der üblichen Standards inklusive einer individuell zu erwägenden Erweiterung der immunsuppressiven Therapie erfolgen (z. B. im Falle einer Myokarditis).

\section{Fazit und geplante Aktualisierung der Konsensus- empfehlungen}

Die erfolgreiche beschleunigte Zulassung von AVXS-101 in den USA ermöglicht erstmals in der Medizingeschichte eine Genersatztherapie der SMA bei Kindern unter 2 Jahren. Im Bereich neuromuskulärer Erkrankungen stellt AVXS-101 die erste AAV-mediierte Gentherapie dar. Die SMA weist einen gut charakterisierten Defekt in einem kleinen, ubiquitär exprimierten Gen mit besonderer klinischer Relevanz im ZNS auf und eignet sich somit in besonderem Maße für eine minimal-invasiv applizierbare ZNS-gerichtete AAV-Gentherapie.
Die vergleichsweise kurzen Studienerfahrungen, die noch nicht bekannte Nachhaltigkeit der Einmalapplikation, die noch nicht vollständig bekannten unerwünschten Nebenwirkungen und schließlich auch die medizinhistorisch höchsten Kosten für das Arzneimittel verlangen nach einem definierten Behandlungskonzept. Die Risiken eines allmählichen Wirkungsverlustes und des Auftretens von Malignomen können auf Basis der aktuellen Datenlage nicht abschließend bewertet werden. Ebenso sind die Vergleiche sowohl bez. der Wirkung als auch der Langzeitkosten gegenüber der aktuell verfügbaren Antisense-Oligonukleotidtherapie mit Nusinersen oder dem oralen SplicingModifikator Risdiplam offen. Weitere Studien und eine systematische Nachbeobachtung und Erfassung der behandelten SMA-Patienten im SMArtCAREoder äquivalenten, industrieunabhängigen Registern müssen das Potenzial und die Risiken der Gentherapie über längere Beobachtungszeiträume evaluieren. Die AAV-Gentherapie ist konzeptionell auf eine einzige intravenöse Anwendung beschränkt. Prinzipiell muss daher auch die Möglichkeit von Kombinationstherapien und einem Therapiewechsel diskutiert werden, um im besten Fall einen zusätz- 
lichen Nutzen zu erzielen. Der Erfolg der neuen Therapien bei der SMA hängt nach den bisherigen Erfahrungen maßgeblich vom Zeitpunkt des Therapiebeginns bzw. Krankheitsstadium ab. Der maximale Therapieerfolg kann nach der aktuellen Datenlage nur in einem präsymptomatischen Stadium der SMA erzielt werden. Die Einführung eines flächendeckenden genetischen Neugeborenenscreenings zur frühzeitigen Erkennung und Behandlung der SMA wird aktuell auf Bundesebene diskutiert. Die Durchführbarkeit einer genetischen Hochdurchsatzuntersuchung als Grundlage einer zukünftigen Aufnahme der SMA in das nationale Neugeborenenscreeningpanel bestätigen erste Ergebnisse eines deutschen Pilotprojektes [14]. Durch die Möglichkeiten der kausalen Therapie der SMA werden "neue“ Phänotypen entstehen, ggf. mit prolongiertem Überleben und neuen Komorbiditäten. Dies erfordert eine besondere Expertise in der interdisziplinären Patientenversorgung und der Transition der SMA-Patienten, die nur von wenigen Kompetenzzentren aufgebracht werden kann. Dieses Papier ist das Ergebnis eines Konsensusprozesses von in der Betreuung und Behandlung von Kindern und Jugendlichen mit SMA erfahrenen Neuropädiatern. Es werden die strukturellen und personellen Voraussetzungen definiert, um den Behandlungserfolg zu überwachen, unerwünschte Wirkungen frühzeitig zu erfassen und die Finanzierung nicht nur des Arzneimittels, sondern auch der Folgekosten zu dokumentieren. Das Konsensuspapier stellt eine Empfehlung dar, wie Kinder mit SMA nach Zulassung durch die EMA in Deutschland mit AVXS-101 behandelt und betreut werden sollen. Es ist vorgesehen, das Konsensuspapier ca. 6 Monate nach Markteinführung der Genersatztherapie im Rahmen einer erneuten Sitzung der Vertreter der pädiatrischen neuromuskulären Behandlungszentren innerhalb des medizinisch-wissenschaftlichen Beirates der DGM weiterzuentwickeln und zu aktualisieren. Unabhängig von diesen Empfehlungen sind ab dem Zeitpunkt der Zulassung die Vorgaben der Fachinformation, der EMA und ggf. des Gemeinsamen Bundesausschusses (G-
BA) zur qualitätsgesicherten Anwendung zu beachten.

\section{Fazit für die Praxis}

- Die neue Genersatztherapie der spinalen Muskelatrophie mit Onasemnogene Abeparvovec hat das Potenzial, den Krankheitsverlauf signifikant positiv und nachhaltig zu beeinflussen.

- Der Erfolg der Therapie hängt nach den bisherigen Erkenntnissen genauso wie bei der zugelassenen Nusinersentherapie vor allem vom Zeitpunkt der Behandlung und damit vom Krankheitsstadium ab. Eine altersgerechte motorische Entwicklung ist allenfalls bei einer präsymptomatisch begonnen Therapie möglich.

- Die Anwendung der Genersatztherapie der SMA muss in spezialisierten Behandlungszentren und unter strengen Sicherheitsauflagen erfolgen, definierte Qualitätskriterien sind einzuhalten.

- Der Erfolg der Behandlung und die Erfassung möglicher Nebenwirkungen müssen systematisch, im Langzeitverlauf und im Vergleich zu anderen Therapien, insbesondere der Nusinersentherapie, in einem nationalen, industrieunabhängigen Register erfasst, dokumentiert und ausgewertet werden. In Deutschland steht mit SMArtCARE eine entsprechende Registerplattform bereits zur Verfügung.

- Die Genersatztherapie der SMA steht exemplarisch für zahlreiche neue und innovative Gentherapeutika, deren erfolgreiche Anwendung in der klinischen Praxis neue Versorgungsstrukturen und eine adäquate Vergütung der Behandlungszentren erfordert.

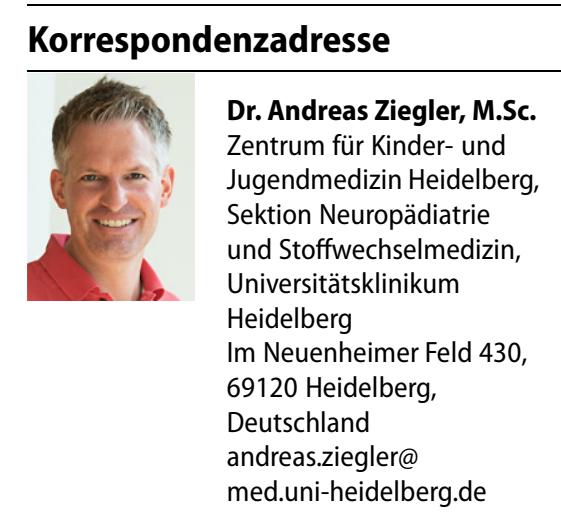

Funding. Open Access funding provided by Projekt DEAL.

\section{Einhaltung ethischer Richtlinien}

Interessenkonflikt. A. Ziegler hat an wissenschaftlichen Veranstaltungen teilgenommen, die von den Firmen Biogen und Sarepta Therapeutics gesponsert wurden. Er ist als Berater für Advisory Boards der Firmen PTC, Avexis, Biogen und Roche in Dienstaufgabe tätig. Zudem war er als Referent für Industriesymposien der Firmen Biogen und Avexis eingeladen ohne Honorarauszahlung auf private Konten. Diese Firmen stellen Therapien für neuromuskuläre Erkrankungen her. E. Wilichowski hat im Rahmen von Kongressen an Workshops teilgenommen, die von den Firmen AveXis, Biogen und Sarepta Therapeutics gesponsert wurden. Er erhielt für Vorträge auf Einladung im Rahmen von Kongressen und Fortbildungsseminaren Honorare und Erstattungen für Reise- und Übernachtungskosten von den Firmen Santhera und Biogen. Er ist als Berater für Advisory Boards der Firmen PTC, Avexis, Biogen und Roche in Dienstaufgabe tätig. U. Schara war als Beraterin für Advisory Boards der Firmen PTC, Avexis, Biogen, Roche, Sarepta, Santhera, Dynacure und Alexion tätig. Zudem war sie als Referentin für Industriesymposien der Firmen Biogen, Avexis, Sarepta, PTC und Santhera eingeladen. Diese Firmen stellen Therapien für neuromuskuläre Erkrankungen her. A. Hahn hat Honorare für Vorträge und Beratungstätigkeit sowie Erstattung von Fortbildungs-, Reise- und Übernachtungskosten der Firmen Biogen; Avexis und Roche erhalten. W. MüllerFelber: Vorträge und Teilnahme an ad hoc Advisory Boards für die Firmen Avexis, Biogen, PTC, Sarepta, Roche. J. Johannsen: Beratertätigkeit bzw. Teilnahme an ad hoc Advisory Boards für die Firmen Avexis, Biogen, PTC, Sarepta, Roche. M. von der Hagen: Honorare für Vorträge und Beratungstätigkeit sowie Erstattung von Fortbildungs-, Reise- und Übernachtungskosten von Biogen, Avexis, Sarepta. A. von Moers hat im Rahmen von Kongressen an Workshops teilgenommen, die von den Firmen Biogen und Sarepta Therapeutics gesponsert wurden. M.C. Walter: Beratertätigkeit und Teilnahme an ad hoc Advisory Boards für die Firmen Avexis, Biogen, Novartis, Pharnext, PTC, Sarepta, Santhera, Roche, Ultragenyx, Wave. R.A. Husain: Honorare für Vorträge und Beratungstätigkeit sowie Erstattung von Fortbildungs-, Reise- und Übernachtungskosten von Biogen; Honorare für Vorträge von PTC. A. Pechmann hat Honorare für Vortragstätigkeiten von Biogen sowie Zuwendungen für klinische Forschungsprojekte von Biogen und Avexis erhalten. J. Kirschner war als Berater und Referent für Avexis, Biogen und Roche 
tätig. Er hat von Avexis, Biogen und Roche Förderung für klinische Studien und Forschungsprojekte erhalten. C. Stoltenburg, A. Saffari, C. Köhler, V. Horber und 0. Schwartz geben an, dass kein Interessenkonflikt besteht.

Für diesen Beitrag wurden von den Autoren keine Studien an Menschen oder Tieren durchgeführt. Für die aufgeführten Studien gelten die jeweils dort angegebenen ethischen Richtlinien.

Open Access. Dieser Artikel wird unter der Creative Commons Namensnennung 4.0 International Lizenz veröffentlicht, welche die Nutzung, Vervielfältigung, Bearbeitung, Verbreitung und Wiedergabe in jeglichem Medium und Format erlaubt, sofern Sie den/die ursprünglichen Autor(en) und die Quelle ordnungsgemäß nennen, einen Link zur Creative Commons Lizenz beifügen und angeben, ob Änderungen vorgenommen wurden.

Die in diesem Artikel enthaltenen Bilder und sonstiges Drittmaterial unterliegen ebenfalls der genannten Creative Commons Lizenz, sofern sich aus der Abbildungslegende nichts anderes ergibt. Sofern das betreffende Material nicht unter der genannten Creative Commons Lizenz steht und die betreffende Handlung nicht nach gesetzlichen Vorschriften erlaubt ist, ist für die oben aufgeführten Weiterverwendungen des Materials die Einwilligung des jeweiligen Rechteinhabers einzuholen.

Weitere Details zur Lizenz entnehmen Sie bitte der Lizenzinformation auf http://creativecommons.org/ licenses/by/4.0/deed.de.

\section{Literatur}

1. Aguti S, Malerba A, Zhou H (2018) The progress of AAV-mediated gene therapy in neuromuscular disorders. Expert Opin Biol Ther 18:681-693

2. Al-Zaidy SA, Mendell JR (2019) From clinical trials to clinical practice: practical considerations for gene replacement therapy in SMA type 1. Pediatr Neurol 100:3-11

3. Chaytow $\mathrm{H}$, Huang YT, Gillingwater $\mathrm{TH}$ et al (2018) The role of survival motor neuron protein $(\mathrm{SMN})$ in protein homeostasis. Cell Mol Life Sci 75:3877-3894

4. Finkel RS, Mercuri E, Meyer $\mathrm{OH}$ et al (2018) Diagnosis and management of spinal muscular atrophy: Part 2: Pulmonary and acute care; medications, supplements and immunizations; other organ systems; and ethics. Neuromuscul Disord 28:197-207

5. Foust KD, Wang X, Mcgovern VL et al (2010) Rescue of the spinal muscular atrophy phenotype in a mouse model by early postnatal delivery of SMN Nat Biotechnol 28:271-274

6. Hinderer C, Katz N, Buza EL et al (2018) Severe toxicity in nonhuman primates and piglets following high-dose intravenous administration of an adeno-associated virus vector expressing human SMN. Hum Gene Ther 29:285-298

7. Louis Jeune V, Joergensen JA, Hajjar RJ et al (2013) Pre-existing anti-adeno-associated virus antibodies as a challenge in AAV gene therapy. Hum Gene Ther Methods 24:59-67

8. Mendell JR, Al-Zaidy S, Shell R et al (2017) Single-dose gene-replacement therapy for spinal muscular atrophy. N Engl J Med 377:1713-1722
9. Mercuri E, Finkel RS, Muntoni F et al (2018) Diagnosis and management of spinal muscular atrophy: Part 1: Recommendations for diagnosis, rehabilitation, orthopedic and nutritional care. Neuromuscul Disord 28:103-115

10. Nault JC, Datta S, Imbeaud S et al (2015) Recurrent AAV2-related insertional mutagenesis in human hepatocellular carcinomas. Nat Genet 47:1187-1193

11. Pechmann A, Konig K, Bernert $G$ et al (2019) SMArtCARE-A platform to collect real-life outcome data of patients with spinal muscular atrophy. Orphanet JRare Dis 14:18

12. Rosas LE, Grieves JL, Zaraspe K et al (2012) Patterns of scAAV vector insertion associated with oncogenic events in a mouse model for genotoxicity. Mol Ther 20:2098-2110

13. Saffari A, Weiler M, Hoffmann GF et al (2019) Gene therapies for neuromuscular diseases. Nervenarzt 90:809-816

14. Vill K, Kolbel H, Schwartz O et al (2019) One year of newborn screening for SMA-Results of a German pilot project. J Neuromuscul Dis 6:503-515

15. Wang D, Tai PWL, Gao G (2019) Adeno-associated virus vector as a platform for gene therapy delivery. NatRev Drug Discov 18:358-378

16. Yong J, Moffett M, Lucas S (2019) Implementing a global Expanded Access Program (EAP) for infantile-onset spinal muscular atrophy (type I): understanding the imperative, impact and challenges. J Neuromuscul Dis 6:227-231

17. Ziegler A, Müller-Felber W, Hahn A, von Moers A, Schara U, Kirschner J (2019) Spinale Muskelatrophie: Gentherapie ohne Zulassung. Dtsch Arztebl 116(48):A-2232/B-1827/C1778 (https://www.aerzteblatt.de/archiv/211081/ Spinale-Muskelatrophie-Gentherapie-ohneZulassung)

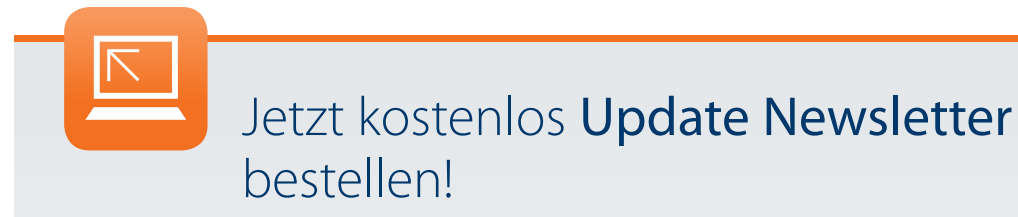

Von Anästhesie bis Urologie - die Update Newsletter von SpringerMedizin.de liefern Ihnen regelmäßig Aktuelles und Wissenswertes aus allen medizinischen Fachgebieten:

- CME-Beiträge aus den Fachzeitschriften von Springer Medizin

- umfassende Übersichtsbeiträge und interessante Kasuistiken

- aktuelle internationale Studien

- Kongress-Highlights und Themen-Specials

- News aus Berufs- und Gesundheitspolitik

Jetzt Newsletter auswählen und kostenlos bestellen unter www.springermedizin.de/mynewsletters 